\title{
Drug-Induced Liver Injury Associated with Lopinavir- Ritonavir in Patients with COVID-19: A Disproportionality Analysis of U.S. Food and Drug Administration Adverse Event Reporting System (FAERS) Data
}

\section{Huilin Tang ( $\nabla$ huiltang85@gmail.com )}

Peking University Health Science Centre https://orcid.org/0000-0002-5814-6657

\section{Liyuan Zhou}

University of North Carolina at Chapel Hill Gillings School of Global Public Health

\section{Xiaotong Li}

Peking University Health Science Centre

\section{Alan C Kinlaw}

University of North Carolina at Chapel Hill Eshelman School of Pharmacy

\section{Jeff $Y$ Yang}

University of North Carolina at Chapel Hill Gillings School of Global Public Health

\section{Andrew M Moon}

University of North Carolina at Chapel Hill School of Medicine

\section{Edward L Barnes}

University of North Carolina at Chapel Hill School of Medicine

\section{Tiansheng Wang}

University of North Carolina at Chapel Hill Gillings School of Global Public Health

\section{Research Article}

Keywords: Lopinavir-ritonavir, Novel coronavirus disease 2019, Liver injury, FAERS

Posted Date: May 11th, 2021

DOI: https://doi.org/10.21203/rs.3.rs-490285/v1

License: (c) (i) This work is licensed under a Creative Commons Attribution 4.0 International License. Read Full License 
Version of Record: A version of this preprint was published at International Journal of Clinical Pharmacy on July 30th, 2021. See the published version at https://doi.org/10.1007/s11096-021-01311-5. 


\section{Abstract \\ Background}

Liver injury has been documented independently in novel coronavirus disease 2019 (COVID-19) patients and patients treated with lopinavir-ritonavir.

\section{Objective}

to investigate the drug-induced liver injury associated with lopinavir-ritonavir among the patients with COVID-19.

\section{Methods}

We conducted a disproportionality analysis of US Food and Drug Administration Adverse Event Reporting System (FAERS) between 2020Q1 and 2020Q3 to evaluate the association between lopinavir-ritonavir and risk of drug-induced liver injury (or severe drug-induced liver injury) and calculated their reporting odds ratios (RORs) with $95 \%$ confidence intervals (Cls).

\section{Results}

A total of 1,754 reports of drug-induced liver injury in patients with COVID-19. The ROR for drug-induced liver injury was $1.4(95 \% \mathrm{Cl}, 1.1-1.7), 3.6(95 \% \mathrm{Cl}, 2.7-4.7)$, and $0.8(95 \% \mathrm{Cl}, 0.7-1.0)$ when comparing lopinavir-ritonavir with all other drugs, hydroxychloroquine/chloroquine only, and remdesivir, respectively. For severe drug-induced liver injury, RORs for lopinavir-ritonavir provided evidence of an association compared with all other drugs (ROR, 4.9; 95\% Cl, 3.7-6.5), compared with hydroxychloroquine/chloroquine only (ROR, 4.3; $95 \% \mathrm{Cl}, 3.0-6.2)$, and compared with remdesivir (ROR, $10.4 ; 95 \% \mathrm{Cl}, 7.2-15.0)$.

\section{Conclusions}

In the FAERS, we observed a disproportional signal for severe drug-induced liver injury associated with lopinavir-ritonavir in patients with COVID-19.

\section{Introduction}

The coronavirus disease 2019 (COVID-19) pandemic has become a global public health crisis. COVID-19 not only produces clusters of severe respiratory illness but also leads to multiorgan failure and death. ${ }^{1}$ Liver injury is common in patients with COVID-19 and is more mommonly observed in severe COVID-19. ${ }^{2}$ 
The mechanism by which COVID-19 may lead to elevated liver enzymes is unclear, but it may be related to several factors including direct virus-induced cytopathic effect, thromboembolic complications, and COVID-19-associated cytokine release ${ }^{2}$. Drug-induced liver injury is another important potential contributor, ${ }^{3}$ but data are limited regarding drug hepatoxicity in patients with COVID-19.

Lopinavir-ritonavir has been used for treating COVID-19 ${ }^{4}$ and is considerated as an independent factor for liver injury, ${ }^{5}$ however, liver injury associated with lopinavir-ritonavir in COVID-19 patients has not been well described. In this study, we investigated the drug-induced liver injury associated with lopinavirritonavir from the US Food and Drug Administration Adverse Event Reporting System (FAERS).

\section{Methods}

We searched FAERS from January 1, 2020 (2020 Q1) to September 30, 2020 (2020 Q3) - all data that were available at submission - to include all reports of COVID-19, using the COVID-19 related terms within Medical Dictionary for Regulatory Activities (MedDRA, version 23.1) (See Table 1). Our outcomes included drug-induced liver injury and severe drug-induced liver injury, which were identified by a narrow Standardized MedDRA Query (SMQ) of "drug related hepatic disorders" and by a narrow SMQ of "drug related hepatic disorders - severe events only", respectively (definitions shown in Table 1). ${ }^{6}$ All drugs of interest were identified through their generic and brand names. We conducted a disproportionality analysis and calculated reporting odds ratios (RORs) with $95 \%$ confidence intervals $(\mathrm{Cl})^{7}$ for the following comparisons: 1) lopinavir-ritonavir versus all other drugs (all drugs except lopinavir-ritonavir used in the COVID-19 cases); 2) lopinavir-ritonavir versus hydroxychloroquine/chloroquine; 3) lopinavirritonavir versus remdesivir. The ROR is calculated by dividing the odds of a liver injury event reported for the drug of interest by the odds of a liver injury event reported for the comparison drugs. We defined a signal of increased risk using a ROR $\geq 2$ with a chi-squared test statistic $\geq 4 .{ }^{8}$ Data analyses were performed using SAS 9.4 (SAS Institute Inc., Cary, NC). 
Table 1

Reporting odds ratio for the lopinavir-ritonavir compared to other drugs in patients with COVID-19a Comparison
No. of liver injury / No. of other ADEs
Lopinavir- Comparator
ritonavir
Reporting odds ratio
$(95 \% \mathrm{Cl})$

Chi-squared test statistic (p-value)

\section{Drug-induced liver injury ${ }^{c}$}

\begin{tabular}{lclll}
$\begin{array}{l}\text { Lopinavir-ritonavir vs all other } \\
\text { drugs }^{\mathrm{b}}\end{array}$ & $132 / 325$ & $\begin{array}{l}1622 / \\
5605\end{array}$ & $\begin{array}{l}1.4(1.1- \\
1.7)\end{array}$ & $10.1(0.002)$ \\
$\begin{array}{lllll}\text { Lopinavir-ritonavir vs } \\
\text { hydroxychloroquine/chloroquine }\end{array}$ & $96 / 212$ & $233 / 1850$ & $\begin{array}{l}3.6(2.7- \\
4.7)\end{array}$ & $90.3(<0.00005)$ \\
\hline Lopinavir-ritonavir vs remdesivir & $131 / 322$ & $\begin{array}{l}1079 / \\
2233\end{array}$ & $\begin{array}{l}0.8(0.7- \\
1.0)\end{array}$ & $2.5(0.12)$
\end{tabular}

\section{Severe drug-induced liver injury ${ }^{d}$}

\begin{tabular}{|c|c|c|c|c|}
\hline $\begin{array}{l}\text { Lopinavir-ritonavir vs all other } \\
\text { drugs }\end{array}$ & $71 / 344$ & 265 / 6319 & $\begin{array}{l}4.9(3.7- \\
6.5)\end{array}$ & $146.2(<0.00005)$ \\
\hline $\begin{array}{l}\text { Lopinavir-ritonavir vs } \\
\text { hydroxychloroquine/chloroquine }\end{array}$ & $51 / 224$ & 99 / 1867 & $\begin{array}{l}4.3(3.0- \\
6.2)\end{array}$ & $70.5(<0.00005)$ \\
\hline Lopinavir-ritonavir vs remdesivir & $71 / 340$ & $58 / 2894$ & $\begin{array}{l}10.4 \\
(7.2- \\
15.0)\end{array}$ & $229.3(<0.00005)$ \\
\hline
\end{tabular}

ADEs, adverse drug events; SMQ, Standardized Medical Dictionary for Regulatory Activities Query.

a MedDRA preferred terms related to COVID-19 including "asymptomatic COVID-19", "COVID-19", "COVID-19 pneumonia", "suspected COVID-19", "SARS-COV-2 carrier", "exposure to SARS-COV-2", "occupational exposure to communicable disease", "occupational exposure to SARS-COV-2", "coronavirus test", "coronavirus test negative", "SARS-COV-2 test", "SARS-COV-2 test false negative", "SARS-COV-2 test negative", "SARS-COV-2 test positive", "COVID-19 prophylaxis", "COVID-19 treatment", "COVID-19 immunisation", "patient isolation", "quarantine", "multisystem inflammatory syndrome in children", "SARS-COV-2 sepsis", "SARS-COV-2 viraemia", "SARS-COV-2 test false positive", "SARS-COV-2 antibody test".

b all other drugs including the all drugs except lopinavir-ritonavir used in the COVID-19 cases.

c identified by a narrow SMQ of "Drug related hepatic disorders," which includes narrow scope of cholestasis and jaundice of hepatic origin, liver related investigations, signs and symptoms, liverrelated coagulation and bleeding disturbances, noninfectious hepatitis, hepatic failure, fibrosis, cirrhosis, other drug-related liver damage, liver neoplasms (benign, including cysts and polyps), and liver neoplasms (malignant and unspecified).

d identified by a narrow SMQ of "Drug related hepatic disorders - severe events only," which includes narrow scope of noninfectious hepatitis, hepatic failure, fibrosis, cirrhosis, other drug-related liver damage, liver neoplasms (benign, including cysts and polyps), and liver neoplasms (malignant and unspecified). 


\section{Results}

A total of 1,754 reports of drug-induced liver injury in patients with COVID-19 were identified within FAERS from 2020 Q1 to 2020 Q3. Of those, 132 reports (7.5\%) were associated with lopinavir-ritonavir use. The results of the disproportionality analysis are presented in Table 1. For lopinavir-ritonavir, $132 /(132+325)$, $28.9 \%$, of reported adverse events were for drug-induced liver injury, compared to $1622 /(1622+5605)$, $22.4 \%$, for all other drugs combined. The ROR for drug-induced liver injury was $1.4(95 \% \mathrm{Cl}, 1.1-1.7)$ when comparing lopinavir-ritonavir with all other drugs. When lopinavir-ritonavir was compared with hydroxychloroquine/chloroquine only, the ROR for drug-induced liver injury was higher, at $3.6(95 \% \mathrm{Cl}$, 2.7-4.7). However, the ROR for drug-induced liver injury comparing lopinavir-ritonavir with remdesivir was small (ROR, 0.8; 95\% Cl, 0.7-1.0).

For severe drug-induced liver injury, RORs for lopinavir-ritonavir provided evidence of an association compared with all other drugs (ROR, 4.9; 95\% $\mathrm{Cl}, 3.7-6.5)$, compared with hydroxychloroquine/chloroquine (ROR, 4.3; 95\% Cl, 3.0-6.2), and compared with remdesivir (ROR, 10.4; $95 \% \mathrm{Cl}, 7.2-15.0)$.

\section{Discussion}

In this disproportionality analysis of FAERS data, we observed increased odds of reporting drug-induced liver injury among patients who used lopinavir-ritonavir for COVID-19. The strongest associations were observed in our analysis of severe drug-induced liver injury, which suggests that patients taking lopinavirritonavir might have a higher risk of severe drug-induced liver injury than those prescribed other COVID-19 therapies.

Our finding that lopinavir-ritonavir might be associated with an increased risk of drug-induced liver injury supports evidence from one recent study. ${ }^{9}$ Lopinavir-ritonavir has been associated with moderate-tosevere elevations in serum aminotransferase levels in prior studies. ${ }^{3,10}$ One retrospective case series in China also demonstrated that a higher proportion of patients with abnormal liver function had taken lopinavir-ritonavir after admission than those with normal liver function (57.8\% vs. $31.3 \%) .{ }^{11}$ The mechanism of lopinavir-ritonavir induced hepatotoxicity may be due to its metabolism by the cytochrome P450 (CYP) system (primarily CYP3A4) in the liver. ${ }^{12}$ Physicians should be aware of the potential risks, including liver injury, when combining lopinavir-ritonavir with the drugs metabolized by CYP450 enzyme (e.g., chloroquine and hydroxychloroquine). ${ }^{13}$ Moreover, recent trials have found that use of lopinavirritonavir did not significantly enhance clinical improvement in adults hospitalized with severe COVID-19.5,

${ }^{14}$ Given these concerns regarding the efficacy and safety of lopinavir-ritonavir in this setting, treatment of COVID-19 with lopinavir-ritonavir may warrant concern given the availability of other therapeutic options.

This study has limitations. Spontaneous events reporting is subject to reporting bias and the lack of denominators to estimate risks of adverse events. Given limited information available in the FAERS database, we cannot control the confounders in our analysis, which include comorbidities, concomitant 
medications, or severity of COVID-19. Although disproportionality analysis is a useful tool for signal detection, causality regarding the association between lopinavir-ritonavir and drug-induced liver injury cannot be established by this study, and further research is needed to supplement our findings.

\section{Conclusions}

In conclusion, we observed a disproportional signal for severe drug-induced liver injury associated with lopinavir-ritonavir in patients with COVID-19, indicating that patients treated with lopinavir-ritonavir may be at increased risk of severe liver injury. Given little evidence of the benefit for the use of lopinavirritonavir in patients with COVID-19 as well as the possible increase in risk for liver injury, other medications might be recommended for routine treatment of COVID-19 instead of lopinavir-ritonavir. Further real-world studies are warranted to evaluate the liver injury risk associated with lopinavir-ritonavir.

\section{Declarations}

\section{Funding None}

\section{Author contributions}

$H T$ and XL designed the study. HT wrote the first draft of the manuscript. LZ did the statistical analysis. TW oversaw and supported programming. HT, AMM, ELB, TW, JYY, and ACK were involved in data review and interpretation. All authors contributed to critical revision of the manuscript for important intellectual content, and approved the final version of the manuscript.

Conflict of interest $\mathrm{HT}$ is a consultant for Evidpro, LLC. All authors have no conflicts of interest that are directly relevant to the content of this article.

\section{References}

1. Huang C, Wang Y, Li X, et al. Clinical features of patients infected with 2019 novel coronavirus in Wuhan, China. Lancet. 2020;395:497-506.

2. Zhang C, Shi L, Wang FS. Liver injury in COVID-19: management and challenges. Lancet Gastroenterol Hepatol. 2020;5:428-30.

3. Olry A, Meunier L, Delire B, Larrey D, Horsmans Y, Le Louet H. Drug-Induced Liver Injury and COVID-19 Infection: The Rules Remain the Same. Drug Saf. 2020.

4. Osborne V, Davies M, Lane S, et al. Lopinavir-Ritonavir in the Treatment of COVID-19: A Dynamic Systematic Benefit-Risk Assessment. Drug Saf. 2020;43:809-21.

5. Cao B, Wang Y, Wen D, et al. A Trial of Lopinavir-Ritonavir in Adults Hospitalized with Severe Covid19. N Engl J Med. 2020;382:1787-99.

6. Gahr M, Zeiss R, Lang D, Connemann BJ, Hiemke C, Schonfeldt-Lecuona C. Drug-Induced Liver Injury Associated With Antidepressive Psychopharmacotherapy: An Explorative Assessment Based on 
Quantitative Signal Detection Using Different MedDRA Terms. J Clin Pharmacol. 2016;56:769-78.

7. Rothman KJ, Lanes S, Sacks ST. The reporting odds ratio and its advantages over the proportional reporting ratio. Pharmacoepidemiol Drug Saf. 2004;13:519-23.

8. Almenoff JS, Pattishall EN, Gibbs TG, DuMouchel W, Evans SJ, Yuen N. Novel statistical tools for monitoring the safety of marketed drugs. Clin Pharmacol Ther. 2007;82:157-66.

9. Cai Q, Huang D, Yu H, et al. COVID-19: Abnormal liver function tests. J Hepatol. 2020;73:566-74.

10. Sherman KE, Shire NJ, Cernohous P, et al. Liver injury and changes in hepatitis C Virus (HCV) RNA load associated with protease inhibitor-based antiretroviral therapy for treatment-naive HCV-HIVcoinfected patients: lopinavir-ritonavir versus nelfinavir. Clin Infect Dis. 2005;41:1186-95.

11. Fan Z, Chen L, Li J, et al. Clinical Features of COVID-19-Related Liver Functional Abnormality. Clin Gastroenterol Hepatol. 2020;18:1561-6.

12. Nunez M. Hepatotoxicity of antiretrovirals: incidence, mechanisms and management. J Hepatol. 2006;44:132-9.

13. Schrezenmeier E, Dorner T. Mechanisms of action of hydroxychloroquine and chloroquine: implications for rheumatology. Nat Rev Rheumatol. 2020;16:155-66.

14. Group RC. Lopinavir-ritonavir in patients admitted to hospital with COVID-19 (RECOVERY): a randomised, controlled, open-label, platform trial. Lancet. 2020. 